\title{
Trastuzumab- and Fab' fragment-modified curcumin PEG-PLGA nanoparticles: preparation and evaluation in vitro and in vivo
}

This article was published in the following Dove Press journal:

International Journal of Nanomedicine

\author{
Dongyu Duan ${ }^{1, *}$ \\ Aiping Wang ${ }^{1, *}$ \\ Ling $\mathrm{Ni}^{2}$ \\ Liping Zhang' \\ Xiuju Yan' \\ Ying Jiang' \\ Hongjie $\mathrm{Mu}^{\prime}$ \\ Zimei Wu' \\ Kaoxiang Sun' \\ Youxin $\mathrm{Li}^{1,2}$
}

'School of Pharmacy, Collaborative Innovation Center of Advanced

Drug Delivery System and Biotech

Drugs in Universities of Shandong,

Key Laboratory of Molecular

Pharmacology and Drug Evaluation

(Yantai University), Ministry of

Education, Yantai University, Yantai,

People's Republic of China; ${ }^{2}$ State

Key Laboratory of Long Acting and

Targeting Drug Delivery System,

Shandong Luye Pharmaceutical Co., Ltd, Yantai, People's Republic of China

*These authors contributed equally to this work
Introduction: Nanoparticles (NPs) modified with bio-ligands represent a promising strategy for active targeted drug delivery to tumour. However, many targeted ligands, such as trastuzumab (TMAB), have high molecular weight, limiting their application for targeting. In this study, we prepared Fab' (antigen-binding fragments cut from TMAB)-modified NPs (Fab'-NPs) with curcumin (Cur) as a model drug for more effective targeting of human epidermal growth factor receptor 2 (HER2/ErbB2/Neu), which is overexpressed on breast cancer cells.

Material and methods: The release kinetics was conducted by dialysis bags. The ability to kill HER2-overexpressing BT-474 cells of Fab'-Cur-NPs compared with TMAB-Cur-NPs was conducted by cytotoxicity experiments. Qualitative and quantitative cell uptake studies using coumarin-6 (fluorescent probe)-loaded NPs were performed by fluorescence microscopy and flow cytometry. Pharmacokinetics and biodistribution experiments in vivo were assessed by liquid chromatography-tandem mass spectrometry (LC-MS/MS).

Results: The release kinetics showed that both Fab'-Cur-NPs and TMAB-Cur-NPs provided continuous, slow release of curcumin for $72 \mathrm{~h}$, with no significant difference. In vitro cytotoxicity experiments showed that Fab'-Cur-NPs manifested prominent ability to kill HER2-overexpressing BT-474 cells compared with TMAB-Cur-NPs. Qualitative and quantitative cell uptake studies indicated that the accumulation of Fab'-NPs was greater than that of TMAB-NPs in BT-474 (HER2+) cells; However, there was no significant difference in MDA-MB-231 (HER2-) cells. Pharmacokinetics and biodistribution experiments in vivo demonstrated that the half-life $(\mathrm{t} 1 / 2)$ and area under the blood concentration-time curve (AUC0-t) of Fab'-Cur-NPs increased 5.30fold and 1.76-fold relative to those of TMAB-Cur-NPs, respectively. Furthermore, the tumor accumulation of Fab'-Cur-NPs was higher than that of TMAB-Cur-NPs.

Conclusion: Fab' fragment has greater capacity than the intact antibody to achieve tumor targeting through NP-based delivery.

Keywords: trastuzumab-modified curcumin nanoparticles, Fab'-modified curcumin nanoparticles, pharmacokinetics, biodistribution, tumour targeting, breast cancer

\section{Introduction}

Conventional anticancer drugs result in many serious side effects owing to unselective distribution and toxicity of the drugs to normal cells. Ideal strategies for the development of anticancer drugs include targeted delivery to tumors, minimal adverse reactions, and high efficacy. Currently, nano-drug delivery systems in the field of oncology are attracting significant interest. ${ }^{1}$ As a new generation of drug delivery systems, nanoparticles (NPs) can increase the delivery of small molecule anticancer drugs to solid tumours. ${ }^{2}$ Drugloaded NPs with a diameter in the range of $200 \mathrm{~nm}$ are deposited in solid tumors because
Correspondence: Youxin Li; Kaoxiang Sun

School of Pharmacy, Yantai University, No 30 Qingquan Road, Yantai, 264005, Shandong Province, People's Republic of China Tel +865353946 400 (Sun) Email youxinli@luye.cn; sunkx@ytu.edu.cn 
of enhanced permeability and retention, which occurs through passive targeting. ${ }^{3}$ In contrast, active targeted drug delivery can be achieved through covalent conjugation, on the nanocontainer surface, of ligands or antibodies that can identify and bind to specific receptors that are overexpressed in cancer cells. ${ }^{4}$

Among many antigens found in cancer cells, human epidermal growth factor receptor 2 (HER2), a member of the EGFR1 family, is highly overexpressed in $25 \%-40 \%$ of human breast cancer cells., 5

Trastuzumab (Herceptin ${ }^{\circledR}$, TMAB), a humanized monoclonal antibody (mAb), which contains two antigen-specific sites that directly bind to the HER2 extracellular domain, ${ }^{7-9}$ provides an admirable strategy for drug targeting to breast cancer cells. ${ }^{10}$ Systematic in vivo experiments have shown that the size of the antibody molecule is a significant parameter in its pharmacokinetics (PK) and biodistribution (BD). ${ }^{11}$ Large TMAB molecule (185 kDa) has been found to penetrate solid tumors slowly, distribute nonuniformly at its final site, and have high serum level-associated toxicities. ${ }^{12}$ Furthermore, whole antibody with fragment crystallizable $(\mathrm{Fc})$ region can increase the recognition of TMAB-modified NPs by the reticuloendothelial system. This effect can be markedly reduced by using antigen-binding fragment (Fab') molecules. ${ }^{13}$ Previous studies have shown that small fragment-modified multifunctional NPs are easily distributed inside cells and even close to the nucleus, whereas TMAB-modified NPs were found only in the proximity of the cell surface. ${ }^{14-16}$ Moreover, the coupling efficiency of TMAB on the surface of nanovectors was low, probably because of the formation of intermolecular disulfide bonds. ${ }^{17}$

In contrast, small $\mathrm{Fab}^{\prime}$ fragments $(45 \mathrm{kDa})$ containing both $\mathrm{V}_{\mathrm{L}}$ and $\mathrm{V}_{\mathrm{H}}$ domains usually retain the antigen-binding affinity of the parental whole antibody, while manifesting increased $\mathrm{PK}$ for tissue penetration. ${ }^{18}$ Furthermore, it has been shown that Fab'modified solid lipid NPs have longer blood circulation times than intact antibodies (eg, OX26 mAb and HD37mAb). ${ }^{13,19}$

To overcome the defects of TMAB conjugation, we used the small molecule fragment Fab' produced by enzymatic digestion of TMAB as a target to ligand, polyethylene glycolmodified poly (lactic-co-glycolic acid) (PEG-PLGA) as the carrier, and curcumin (Cur) as a model drug to prepare immunized NPs for more effective targeting of HER2-overexpressing cells. Experiments were conducted both in vitro and in vivo to evaluate the advantages of these Fab'-modified NPs for targeting and penetrating tumor tissues compared to TMAB-NPs.

\section{Materials and methods}

\section{Materials}

2-Iminothiolane (Traut's reagent) was purchased from SigmaAldrich Co. (St Louis, MO, USA). TMAB was purchased from Hoffman-La Roche Ltd. (Basel, Switzerland). PEGPLGA-maleimide (mal-PEG-PLGA; 5,000-20,000 Da, 50:50 LA:GA, w/w) and methoxy-PEG-PLGA (mPEG-PLGA; 5,000-20,000 Da, 50:50 LA:GA, w/w) were purchased from Polyscitech (West Lafayette, IN, USA). Furthermore, 3-(2'benzothiazolyl)-7-diethylaminocoumarin (coumarin-6) was purchased from Aladdin Industrial Corp. (Shanghai, China). A bicinchoninic acid (BCA) protein quantitation kit was purchased from Beyotime Biotechnology (Shanghai, China). Cur was purchased from Sinopharm Chemical Reagent Co. Ltd. (Beijing, China). All reagents were of laboratory grade.

\section{Cell lines, antibodies, and animals}

The HER2 ${ }^{+}$breast cancer cell line (BT-474) and the HER2breast cancer cell line (MDA-MB-231) were purchased from Cobioer (Nanjing, People's Republic of China).

$\mathrm{F}\left(\mathrm{ab}^{\prime}\right)_{2}$ fragments of TMAB were produced by the digestion of TMAB using immobilized pepsin, according to the manufacturer's protocol. The disulfide bonds in $\mathrm{F}\left(\mathrm{ab}^{\prime}\right)_{2}$, were reduced with $20 \mathrm{mM}$ cysteine to produce $\mathrm{Fab}^{\prime}$, which contains one terminal free thiol group per $\mathrm{Fab}^{\prime}$ molecule, for use in conjugation protocols.

Female BALB/c mice of 6-8 weeks were purchased from Charles River Laboratories (Beijing Vital River Laboratory Animal Technology Co., Ltd, Beijing, People's Republic of China). Sprague-Dawley rats (180-200 g) were kindly provided by the Luye Pharma Group (Shandong, People's Republic of China). All animal studies were performed according to the Guide for the Care and Use of Laboratory Animals. The Animal Ethics Committee of Yantai University approved the study.

\section{Preparation of NPs}

The single-emulsion technique was used to prepare NPs. ${ }^{20}$ Briefly, mPEG-PLGA, mal-PEG-PLGA, and Cur or coumarin- 6 were weighed and dissolved in the organic phase $(2: 1 \mathrm{v} / \mathrm{v}$ dichloromethane/acetone). The solution was then added to a polyvinyl alcohol (PVA) solution $(2 \% \mathrm{w} / \mathrm{v})$ drop by drop with continuous vortexing. Next, the mixture was sonicated intermittently using a probe sonicator (output power of $550 \mathrm{~W}, 8$ times) on ice to create an oil-in-water emulsion. This emulsion was then added to a PVA $(0.5 \% \mathrm{w} / \mathrm{v})$ solution with magnetic stirring. Cur and coumarin-6 NPs were collected by centrifugation at 5,000 rpm for 30 minutes and washed three times in Milli-Q water. ${ }^{21-23}$

TMAB- and Fab'-modified NPs were prepared using a postinsertion method. ${ }^{24}$ TMAB and $\mathrm{Fab}^{\prime}$ were covalently attached to the outer membrane of the NPs prepared using the above method (Cur or coumarin-6 NPs) by thioether bonds formed by the reaction of maleimide with free sulfhydryl groups. 
To generate free sulfhydryl groups, TMAB was thiolated with 2-iminothiolane (Traut's reagent) at a molar ratio of 1:20 (TMAB:Traut's reagent) in PBS (pH 8.0) for 60 minutes. After thiolation, unreacted Traut's reagent was removed via Sephadex G-25 columns. ${ }^{1}$ Compared to TMAB, Fab' fragments bear sulfhydryl groups that are used directly. ${ }^{13}$ To generate Fab'-NPs and TMAB-NPs, Fab' fragments and thiolated TMAB were mixed with maleimide-functionalized NPs and incubated at room temperature for 16 hours. Considering each TMAB contains two HER2 binding domains, the $\mathrm{Fab}^{\prime}$ fragments and thiolated TMAB were coupled to NPs with a molar ratio of 1:10 (Fab':Mal-PEG-PLGA) or 1:20 (TMAB:Mal-PEG-PLGA). Unconjugated protein was removed using Sepharose CL-4B columns. Protein conjugation efficiency was confirmed with the BCA kit.

\section{Characterization of NPs}

Morphology, particle size, and encapsulation efficiency (EE)

Morphological examination of NPs was performed by transmission electron microscopy (TEM, H-600; Hitachi, Tokyo, Japan). The mean particle size of NPs was assessed using a particle analyser (Delsa Nano C; Beckman Coulter Inc., Brea, CA, USA).

EE was assessed by ultrafiltration. Samples were loaded into an ultrafiltration device (100 kMWCO; Sartorius, Göettingen, Germany) and centrifuged at 5,000 rpm for 30 minutes at $4^{\circ} \mathrm{C}$ to remove any free drug. The same volume of each sample was dissolved in acetonitrile to confirm the total amount of drug. The concentration was measured by high-performance liquid chromatography (HPLC). For Cur, isocratic elution was conducted at a flow rate of $1 \mathrm{~mL} / \mathrm{min}$ using a 40:60 mixed solution of mobile phase $\mathrm{A}(0.5 \%$ glacial acetic acid [HAC] in Milli-Q water) and mobile phase B (acetonitrile). The samples were monitored at $425 \mathrm{~nm}$. For coumarin-6, isocratic elution was conducted at a flow rate of $1 \mathrm{~mL} / \mathrm{min}$ using a 4:96 mixed solution of mobile phase A (Milli-Q water) and mobile phase B (methanol). The excitation and emission wavelengths were 466 and $504 \mathrm{~nm}$, respectively. Drug EE (\%) of the NPs was calculated using the following equation:

$$
\mathrm{EE}(\%)=\frac{\text { Amount of drug entrapped }}{\text { Total amount of drug }} \times 100 \%
$$

\section{In vitro drug release study}

In vitro drug release studies were conducted using dialysis bags with a molecular weight cutoff of $10 \mathrm{kDa}$. The release profiles for Cur-loaded NPs were measured at $37^{\circ} \mathrm{C}$ in $200 \mathrm{~mL}$ of $0.1 \mathrm{M}$ phosphate-buffered saline (PBS; pH 5.8) for 144 hours. The concentration of Cur in the samples was detected by HPLC as described above. All samples were analyzed in triplicate, and the results were presented as mean \pm standard deviation (SD).

\section{In vitro studies}

\section{In vitro cellular uptake}

In the subsequent studies, coumarin-6, a fluorescent probe, was loaded into NPs having the same particle size as Curloaded NPs to examine the targeting of NPs.

Quantitative analyses of cellular uptake of coumarin-6 solution, coumarin-6-labeled NPs, TMAB-NPs, and Fab'NPs were performed by flow cytometry (BD Accuri C6; BD Biosciences, San Jose, CA, USA). Qualitative analyses were conducted by fluorescence microscopy (Vert.A1; Carl Zeiss AG, Oberkochen, Germany).

For flow cytometry, BT-474 (HER2 ${ }^{+}$) cells and MDAMB-231 (HER2-) cells in the logarithmic phase were seeded into six-well plates at a density of $1 \times 10^{5}$ cells per well, followed by incubation at $37^{\circ} \mathrm{C}$ in $5 \% \mathrm{CO}_{2}$. After 48 hours, the cells were treated with coumarin- 6 solution, TMABNPs, Fab'-NPs, or NPs with increasing concentrations (1-10 ng/mL) of coumarin- 6 for 1.5 hours. In addition, the cells were incubated with coumarin-6 solution, TMAB-NPs, Fab'-NPs, or NPs containing coumarin-6 $(5 \mathrm{ng} / \mathrm{mL})$ for 0.25 , $0.75,1.25$, or 2 hours, respectively. Subsequently, the cells were washed thrice with cold PBS, trypsinized, and centrifuged at 1,500 rpm for 5 minutes. Finally, the cells were resuspended in PBS and analyzed by flow cytometry.

For fluorescence microscopy, BT-474 (HER2 ${ }^{+}$) cells and MDA-MB-231 (HER2-) cells in the logarithmic phase were seeded into 24-well plates at a density of 3,000 cells per well followed by incubation at $37^{\circ} \mathrm{C}$ in $5 \% \mathrm{CO}_{2}$. After 48 hours, the cells were incubated with coumarin- 6 solution, TMABNPs, Fab'-NPs, or NPs containing coumarin-6 (200 ng/mL) for 5 minutes, $0.5,1$, or 2 hours. The cells were then washed thrice with cold PBS and fixed with 4\% paraformaldehyde for $15 \mathrm{~min}$. The cells were again washed thrice with cold PBS and visualized by fluorescence microscopy.

\section{Intracellular localization of NPs}

BT-474 (HER2 ${ }^{+}$) cells in the logarithmic phase were seeded into 24-well plates containing glass coverslips at a density of 5,000 cells per well. After 48 hours, the cells were incubated with TMAB-NPs, Fab'-NPs, or NPs containing coumarin-6 $\left(200 \mathrm{ng} / \mathrm{mL}\right.$ ) for $0.5,1$, or 2 hours at $37^{\circ} \mathrm{C}$ in $5 \% \mathrm{CO}_{2}$. The cells were washed with cold PBS three times and fixed with 
4\% formaldehyde for 10 minutes. To label the cell nucleus for locating NPs within cells, cell nuclei were counterstained with $5 \mu \mathrm{g} / \mathrm{mL}$ Hoechst 33342 for 15 minutes. Confocal images were obtained by confocal microscopy (Olympus FV1000; Olympus Corporation, Tokyo, Japan).

\section{In vitro cytotoxicity assay}

The cytotoxicity of free Cur, Cur-NPs, TMAB-Cur-NPs, and Fab'-Cur-NPs was determined using an MTT assay. BT-474 $\left(\right.$ HER $\left.^{+}\right)$cells in the logarithmic phase were seeded into a 96-well plate at a density of 4,000 cells per well followed by incubation for 48 hours at $37^{\circ} \mathrm{C}$ in $5 \% \mathrm{CO}_{2}$. The cells were then treated with Cur, Cur-NPs, TMAB-Cur-NPs, or $\mathrm{Fab}^{\prime}$-Cur-NPs with increasing concentrations of Cur $(3,6,12$, 24 , or $50 \mu \mathrm{g} / \mathrm{mL}$ ) for 48 hours at $37^{\circ} \mathrm{C}$ in $5 \% \mathrm{CO}_{2}$. Twenty microliters of MTT solution $(5 \mathrm{mg} / \mathrm{mL})$ was then added into each well and incubated for 4 hours. Subsequently, to solubilize the resultant formazan crystals, $200 \mu \mathrm{L}$ of dimethyl sulfoxide was added into each well followed by shaking for 10 minutes. Finally, the absorbance of each well was measured at $570 \mathrm{~nm}$.

\section{In vivo $P K$ and $B D$ of Cur-NPs, TMAB-Cur-NPs, and Fab'-Cur-NPs}

Sprague-Dawley rats weighing 180-200 g were used for PK studies. The rats were divided into four groups and injected with Cur solution, TMAB-Cur-NPs, Fab'-Cur-NPs, or CurNPs of the equivalent Cur dose $(4 \mathrm{mg} / \mathrm{kg}, \mathrm{n}=5)$ via the tail vein. Blood samples were collected at pre-specified time points $(0.083,0.25,0.5,0.75,1,2,4,6,8,10,12,24$, and 48 hours) postinjection and centrifuged at 3,600 rpm for 10 minutes to obtain plasma.

$\mathrm{BD}$ studies were conducted in heterotopic tumor-bearing BALB/c mice (subcutaneously injected with $2 \times 10^{7}$ BT-474 cells). Mice were injected with TMAB-Cur-NPs, Fab'-CurNPs, or Cur-NPs of the equivalent Cur dose $(4 \mathrm{mg} / \mathrm{kg}$, $\mathrm{n}=3)$ via the tail vein. At selected time points $(0.083,0.25$, $0.5,1,2,4,8,12$, and 24 hours), tumor and major organs (ie, heart, liver, kidneys, spleen, and lung) were excised from euthanized animals. Three volumes of saline were added into tissues to obtain homogenates.

To extract Cur, two volumes of acetonitrile were added to plasma and tissue homogenates. The concentration of Cur was measured via liquid chromatography-tandem mass spectrometry (LC-MS/MS) (AB Sciex Triple Quad ${ }^{\mathrm{TM}} 4500$; Sciex, Framingham, MA, USA) under the following conditions: chromatographic column, Agilent ZORBAX SB-C18
$(100 \times 2.1 \mathrm{~mm}, 5 \mu \mathrm{m})$; mass spectrometer ion source, electrospray ionization source (Cur ion pair, 369.1/285.0).

\section{Statistical analysis}

Data are presented as the mean \pm standard deviation (SD) and were evaluated by analysis of variance with IBM SPSS Statistics 20.0 software (IBM Corporation, Armonk, NY, USA). Pairwise statistical comparisons were performed using a two-tailed Student's $t$-test. Differences were considered statistically significant for a $P$-value $<0.05$ and highly significant for a $P$-value $<0.01$.

\section{Results and discussion \\ Characterization of NPs in vitro}

Cur and coumarin-6 NPs were prepared using the singleemulsion method. TMAB and $\mathrm{Fab}^{\prime}$ fragments were attached to the outer membrane of NPs using a postinsertion method by modification. As shown in Table 1, the size of Cur-NPs, Fab'-Cur-NPs, and TMAB-Cur-NPs was $\sim 120 \mathrm{~nm}$. Particles modified with TMAB or Fab' fragments were $\sim 20 \mathrm{~nm}$ larger as expected, owing to the more complicated surface structure of protein-modified NPs. The particle size of coumarin-6-loaded NPs was approximately the same particle size as those of Cur-loaded NPs (Table 2). The EE of coumarin-6-loaded NPs was lower than that of Curloaded NPs, but with strong fluorescence, and can meet the needs for qualitative and quantitative analysis in vitro cell uptake experiments. The TEM micrograph of Fab'-Cur-NPs (Figure 1A) and TMAB-Cur-NPs (Figure 1B) showed that Cur-NPs were spherical. However, the particle size was smaller than that measured by dynamic light scattering (DLS) (Table 1). The size difference between TEM and DLS measurements was attributed to the state of NPs used for measurements. ${ }^{25}$ The coupling efficiency of TMAB and $\mathrm{Fab}^{\prime}$ on the surface of NPs was $27.7 \% \pm 1.67 \%$ and $64.8 \% \pm 2.32 \%$, respectively. The coupling efficiency of

Table I Characterization of Cur-NPs, Fab'-Cur-NPs, and TMABCur-NPs

\begin{tabular}{llll}
\hline Formulation & $\begin{array}{l}\text { Particle size, } \\
\mathbf{n m}\end{array}$ & $\begin{array}{l}\text { Polydispersity } \\
\text { index }\end{array}$ & EE, \% \\
\hline Cur-NPs & $118.3 \pm 2.3$ & $0.102 \pm 0.038$ & $85.02 \pm 4.2$ \\
Fab'-Cur-NPs & $128.5 \pm 1.3$ & $0.125 \pm 0.012$ & $79.5 \pm 1.56$ \\
TMAB-Cur-NPs & $142.5 \pm 4.6$ & $0.137 \pm 0.023$ & $77.1 \pm 5.64$ \\
\hline
\end{tabular}

Abbreviations: EE, encapsulation efficiency; Cur, curcumin; Cur-NPs, curcumin nanoparticles; Fab'-Cur-NPs, fragment Fab'-modified curcumin nanoparticles; TMAB-Cur-NPs, trastuzumab-modified curcumin nanoparticles. 
Table 2 Characterization of coumarin-6-loaded NPs

\begin{tabular}{llll}
\hline Formulation & $\begin{array}{l}\text { Particle size, } \\
\mathbf{n m}\end{array}$ & $\begin{array}{l}\text { Polydispersity } \\
\text { index }\end{array}$ & EE, \% \\
\hline NPs/coumarin-6 & $\mathrm{I} 12.3 \pm \mathrm{I} .2$ & $0.092 \pm 0.048$ & $75.47 \pm 3.78$ \\
Fab'-NPs/coumarin-6 & $\mathrm{I} 18.5 \pm \mathrm{I} .3$ & $0.109 \pm 0.012$ & $76.14 \pm 5.16$ \\
TMAB-NPs/coumarin-6 & $\mathrm{I} 33.5 \pm 4.6$ & $0.117 \pm 0.013$ & $72.49 \pm 4.67$ \\
\hline
\end{tabular}

Abbreviations: EE, encapsulation efficiency; NPs/coumarin-6, coumarin-6 nanoparticles; Fab'-NPs/coumarin-6, fragment Fab'-modified coumarin-6 nanoparticles; TMAB-NPs/coumarin-6, trastuzumab-modified coumarin-6 nanoparticles.

TMAB was likely to be lower owing to the formation of intermolecular disulfide bonds. ${ }^{17}$

In vitro kinetics of Cur release from the NPs is shown in Figure 2. Free Cur (without NPs) was quickly and almost completely released from the dialysis bag, with $96.4 \% \pm 3.1 \%$ release observed within 8 hours. With regard to the CurNPs, $78.1 \% \pm 10.2 \%, 77 \% \pm 7.5 \%$, and $80 \% \pm 3.4 \%$ of Cur was released from Cur-NPs, Fab'-Cur-NPs, and TMAB-Cur-NPs at 72 hours, respectively, representing a ninefold increase in the time required to reach approximately the same level of drug release as was observed for free Cur. We observed no significant difference in Cur release between Fab'-Cur-NPs and TMAB-Cur-NPs in vitro. These results demonstrate that Cur-loaded NPs exhibit extended release behavior.

\section{Cellular uptake and localization in vitro}

The cellular uptake of coumarin-6 solution, coumarin-6labeled NPs, TMAB-NPs, and Fab'-NPs was evaluated in BT-474 (HER2') and MDA-MB-231 (HER2-) cells by flow cytometry (Figure 3). Following incubation with coumarin-6 solution, TMAB-NPs, Fab'-NPs, or NPs at increasing concentrations $(1-10 \mathrm{ng} / \mathrm{mL})$ or for a single concentration over time
(0.25-2 hours), the mean fluorescence intensities of both cell types increased. This shows that cellular uptake of coumarin-6 solution, TMAB-NPs, Fab'-NPs, and NPs by BT-474 or MDA-MB-231 cells is both time-dependent (Figure 3A and $\mathrm{B}$ ) and concentration-dependent (Figure $3 \mathrm{C}$ and $\mathrm{D}$ ).

Notably, at a given concentration or time-point, TMABNPs and Fab'-NPs were taken up by BT-474 (HER2 ${ }^{+}$) cells significantly more efficiently $(P<0.01)$ than by MDA-MB231 cells. This is likely due to high HER2 receptor expression on the surface of BT- 474 cells, allowing TMAB and Fab' fragments to promote the uptake of target-modified NPs more efficiently via receptor-mediated endocytosis. In addition, the mean fluorescence intensities of BT-474 (HER2 ${ }^{+}$) cells incubated with Fab'-NPs were significantly higher $(P<0.05)$ than those of TMAB-NPs. This shows that small fragment Fab' provides improved tumor cell penetration relative to intact antibody in vitro. ${ }^{11}$ Notably, NPs alone displayed similar cellular uptake by BT-474 and MDA-MB-231 cells, suggesting a HER2-independent mechanism of NP uptake. Furthermore, cellular uptake of coumarin- 6 solution by BT-474 and MDA-MB-231 cells was significantly lower $(P<0.05)$ than that of coumarin-6-loaded NPs. These results were confirmed by fluorescence microscopy as shown in Figure 4.

Intracellular localization of NPs was imaged by confocal microscopy. As shown in Figure 5, coumarin-6-labeled TMAB-NPs, Fab'-NPs, and NPs were observed surrounding the cell nuclei, confirming the uptake of NPs into the cytoplasm of BT-474 cells. Moreover, the accumulation of Fab'-NPs around the nuclei is higher than that of TMABNPs, further indicating that $\mathrm{Fab}^{\prime}$ provides improved tumor cell penetration.
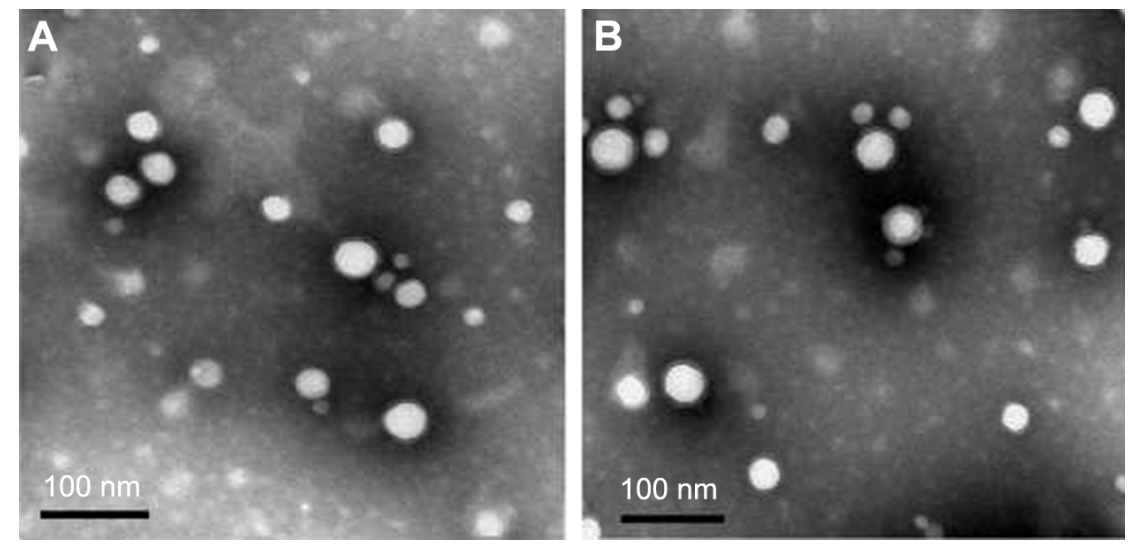

Figure I TEM characterization of Fab'-Cur-NPs (A) and TMAB-Cur-NPs (B).

Abbreviations: TEM, transmission electron microscope; Cur, curcumin; Fab'-Cur-NPs, fragment Fab'-modified curcumin nanoparticles; TMAB-Cur-NPs, trastuzumabmodified curcumin nanoparticles. 


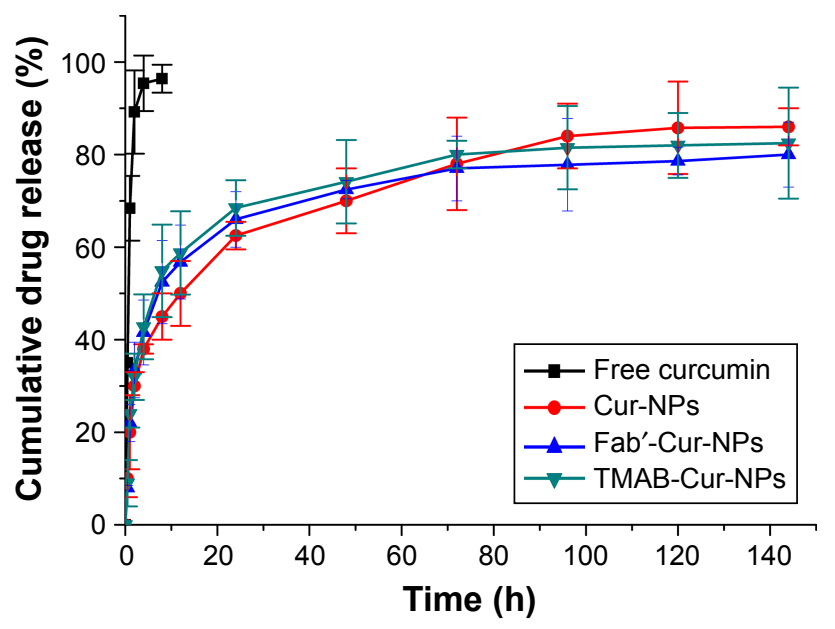

Figure 2 In vitro release studies of curcumin from free curcumin (curcumin solution), Cur-NPs, Fab'-Cur-NPs, and TMAB-Cur-NPs.

Abbreviations: Cur, curcumin; Cur-NPs, curcumin nanoparticles; Fab'-Cur-NPs, fragment Fab'-modified curcumin nanoparticles; TMAB-Cur-NPs, trastuzumabmodified curcumin nanoparticles.
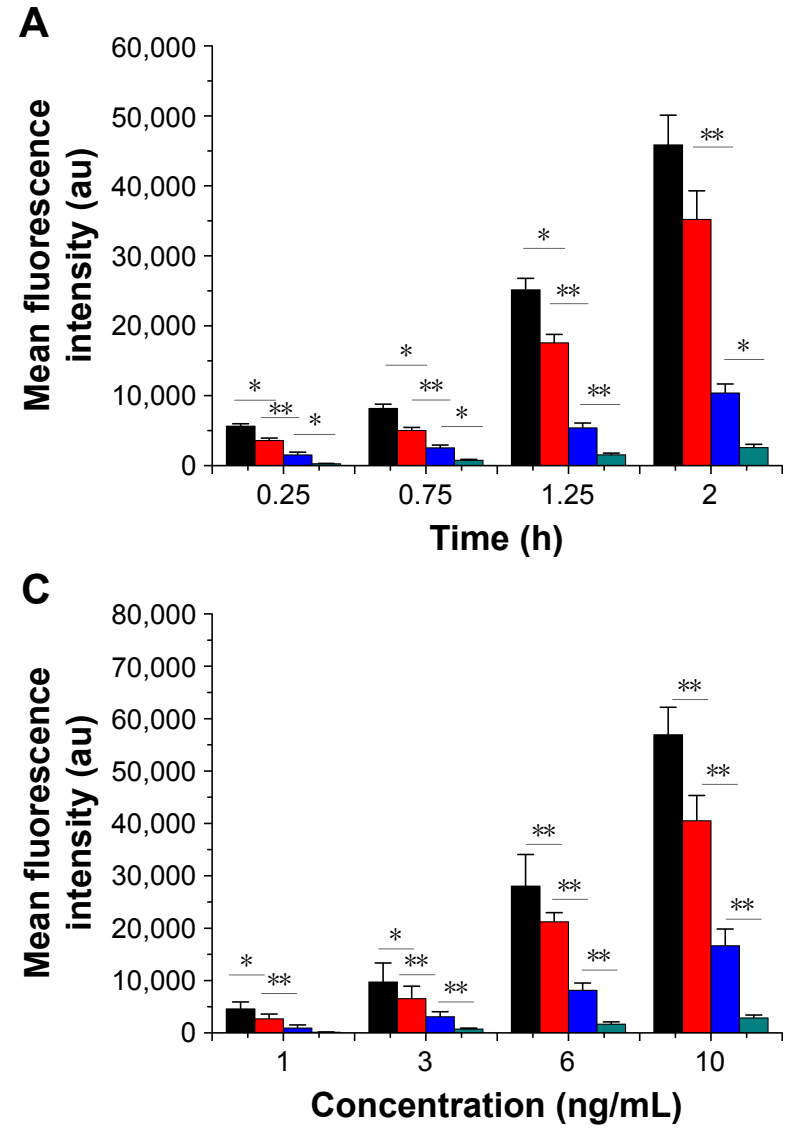

\section{In vitro cytotoxicity}

We next tested the cytotoxic capacity of different formulations against BT-474 (HER2 ${ }^{+}$) cells. Fab'-Cur-NPs, TMABCur-NPs, Cur-NPs, and free Cur solution were incubated with BT-474 cells for 48 hours followed by the evaluation of cell viability by MTT assay. NPs, TMAB-NPs, and $\mathrm{Fab}^{\prime}$-NPs without Cur were nontoxic to BT-474 cells at all concentrations tested (data not shown). Furthermore, as shown in Figure 6, Cur solution, Fab'-Cur-NPs, TMABCur-NPs, and Cur-NPs did not cause severe cell death at lower concentrations (3-6 $\mu \mathrm{g} / \mathrm{mL}$ ). However, exposure to higher concentrations $(12-50 \mu \mathrm{g} / \mathrm{mL})$ of TMAB-Cur-NPs or

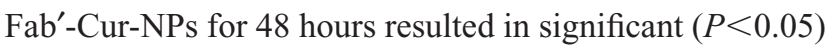
inhibition relative to the same concentration of Cur solution or Cur-NPs, indicating that NPs modified with TMAB and $\mathrm{Fab}^{\prime}$ can be used to efficiently deliver anticancer drugs to
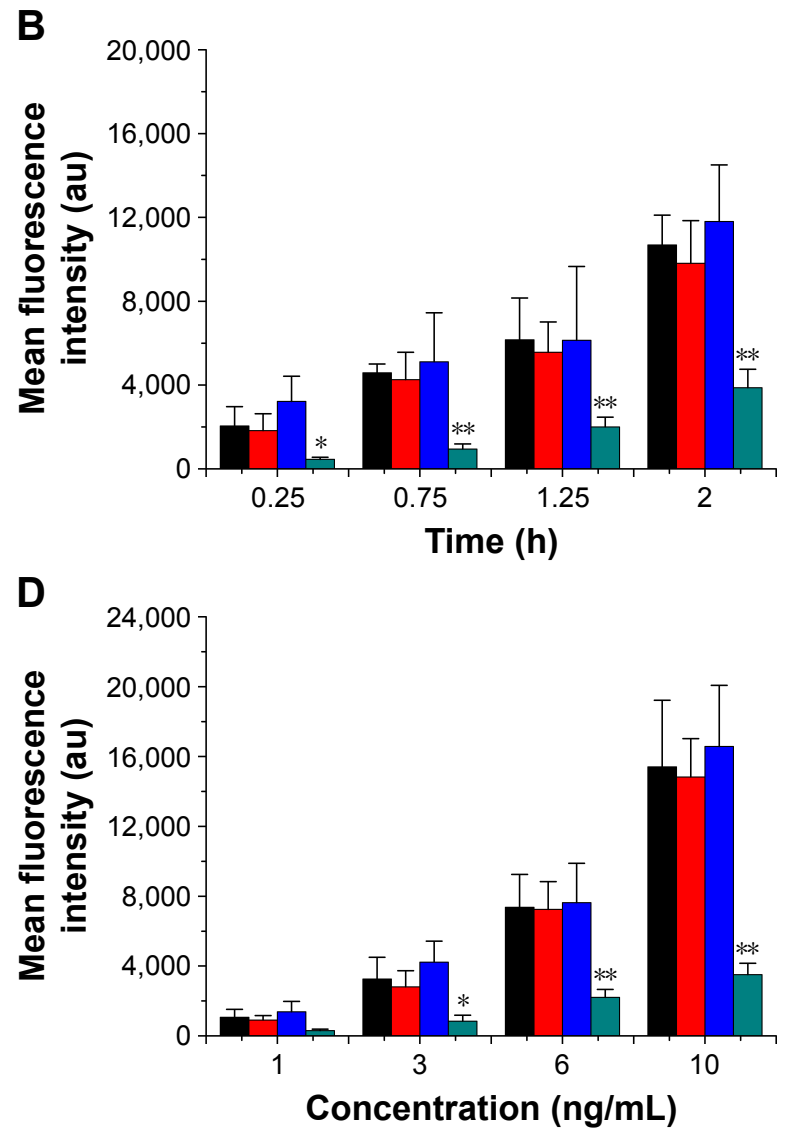

Fab'-NPs $\square$ TMAB-NPs

$\square$ NPs $\square$ Coumarin-6

Figure 3 Quantitative flow cytometry studies of coumarin-6 solution and coumarin-6-labeled TMAB-NPs, Fab'-NPs, and NPs in BT-474 (HER2') cells and MDA-MB-23I (HER2-) cells.

Notes: (A, B) Time-dependent studies of coumarin-6 solution and coumarin-6 labeled TMAB-NPs, Fab'-NPs, and NPs in (A) BT-474 (HER2 ${ }^{+}$) cells and (B) MDA-MB23 I (HER2-) cells; (C, D) Concentration-dependent studies of coumarin-6 solution and coumarin-6 labeled TMAB-NPs, Fab'-NPs, and NPs in (C) BT-474 (HER2 ${ }^{+}$) cells and (D) MDA-MB-23I (HER2-) cells. Significant differences in coumarin-6 solution and coumarin-6-labeled TMAB-NPs, Fab'-NPs, and NPs are marked with * for $P<0.05$ and ** for $P<0.0$ I. Values represent the mean $\pm S D(n=3)$.

Abbreviations: TMAB-NPs, trastuzumab-modified nanoparticles; Fab'-NPs, fragment Fab'-modified nanoparticles; NPs, nanoparticles; HER2, human epidermal growth factor receptor 2 . 
A

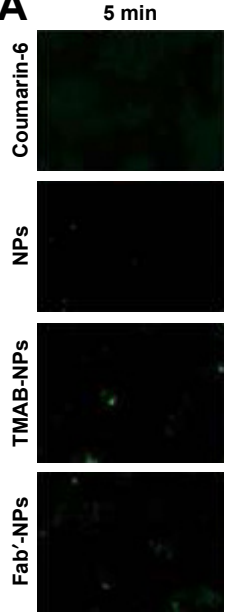

B
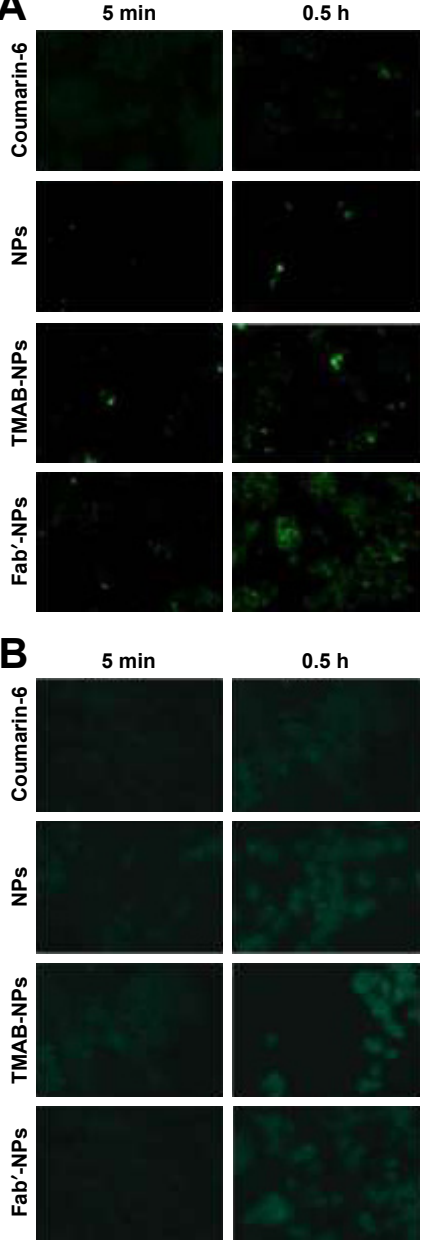

$0.5 \mathrm{~h}$
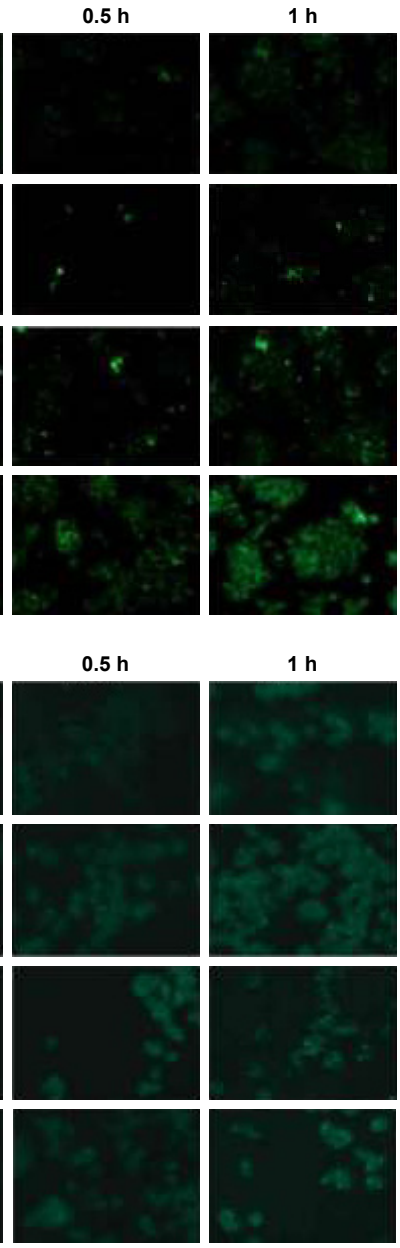

$1 \mathrm{~h}$
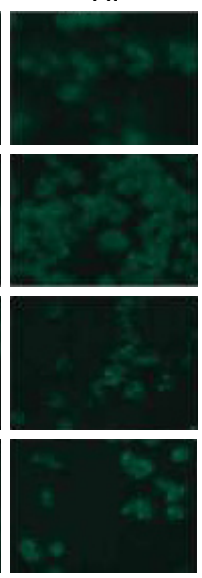

Figure 4 Fluorescence microscopy images of cellular uptake of coumarin-6 solution and coumarin-6-labeled NPs, TMAB-NPs, and Fab'-NPs in BT-474 (HER2 ${ }^{+}$) cells and MDA-MB-23I (HER2-) cells.

Notes: (A, B) Qualitative studies of coumarin-6 solution and coumarin-6-labeled NPs, TMAB-NPs, and Fab'-NPs in (A) BT-474 cells and (B) MDA-MB-23I cells. The scale bar for $\mathbf{A}$ and $\mathbf{B}$ is $20 \mu \mathrm{m}$.

Abbreviations: TMAB-NPs, trastuzumab-modified nanoparticles; Fab'-NPs, fragment Fab'-modified nanoparticles; NPs, nanoparticles; HER2, human epidermal growth factor receptor 2 .

HER2-overexpressing tumor cells. Compared with TMABmodified NPs, Fab'-modified NPs led to significantly greater $(P<0.05)$ growth inhibition in HER2-overexpressing cells, indicating that Fab'-NPs conjugated to a small molecule drug exhibited improved tumor cell penetration as well as anticancer activity in vitro.

\section{PK and BD studies}

The major PK parameters for Cur in Sprague-Dawley rats following tail vein injection of Cur solution or Cur-NPs (CurNPs, Fab'-Cur-NPs, or TMAB-Cur-NPs) are summarized in Table 3. The plasma concentration-time curves for Cur in Sprague-Dawley rats are shown in Figure 7. We observed a significant difference in half-life $\left(\mathrm{t}_{1 / 2}\right)$ and area under the blood concentration-time curve $\left(\mathrm{AUC}_{0-t}\right)$ between Cur

\section{$0.5 \mathrm{~h}$}
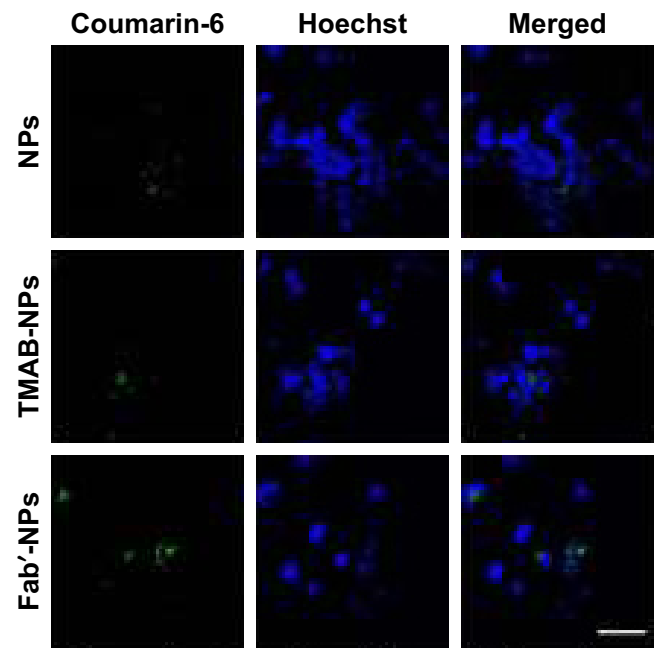

$1 \mathrm{~h}$
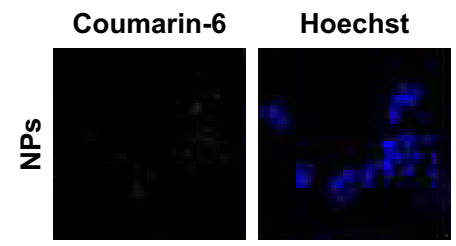

Merged
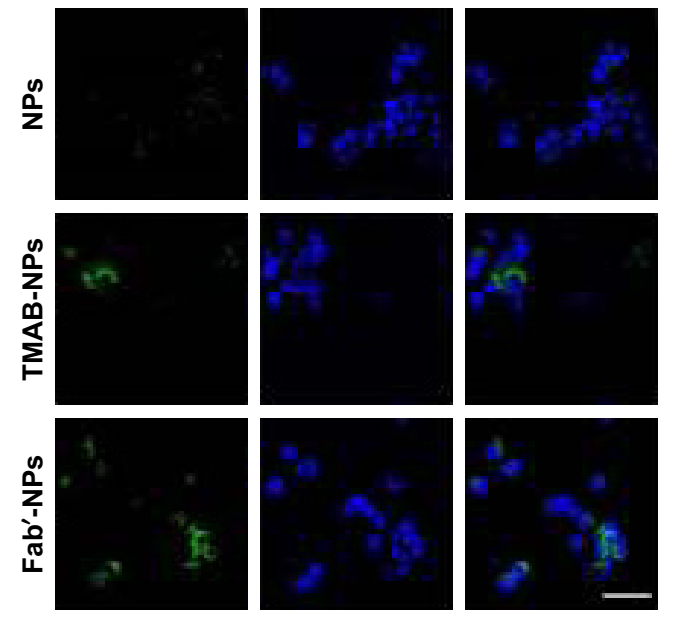

$2 \mathrm{~h}$
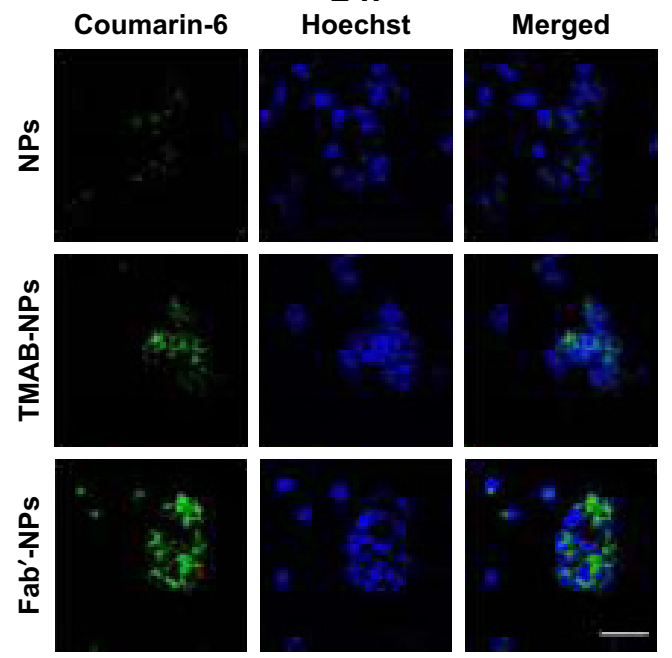

Figure 5 Confocal fluorescence microscopy images of coumarin-6-labeled NPs, TMAB-NPs, and Fab'-NPs bound to BT-474 (HER2 ${ }^{+}$) cells.

Notes: Cell nucleus was visualized using Hoechst 33342 (blue), and coumarin-6labeled NPs (green) were detected in the cytoplasm. The scale bar is $20 \mu \mathrm{m}$. Abbreviations: TMAB-NPs, trastuzumab-modified nanoparticles; Fab'-NPs, fragment Fab'-modified nanoparticles; NPs, nanoparticles; HER2, human epidermal growth factor receptor 2 . 


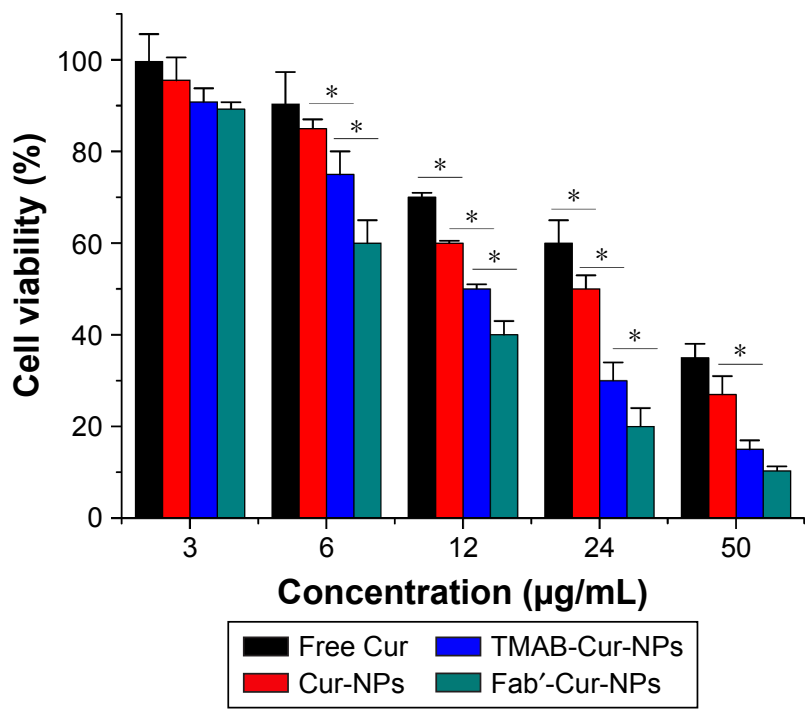

Figure 6 In vitro cytotoxicity of curcumin solution, Cur-NPs, Fab'-Cur-NPs, and TMAB-Cur-NPs on BT-474 (HER2+) cells.

Notes: Significant differences in curcumin solution (free Cur), Cur-NPs, Fab'-CurNPs, and TMAB-Cur-NPs are marked with $*$ for $P<0.05$. Values represent mean \pm $\mathrm{SD}(\mathrm{n}=5)$.

Abbreviations: Cur, curcumin; Cur-NPs, curcumin nanoparticles; Fab'-Cur-NPs, fragment Fab'-modified curcumin nanoparticles; TMAB-Cur-NPs, trastuzumabmodified curcumin nanoparticles; HER2, human epidermal growth factor receptor 2.

solution and Cur-NPs. The $\mathrm{AUC}_{0-\mathrm{t}}$ for Cur-NPs, Fab'-CurNPs, and TMAB-Cur-NPs was 18.36-fold, 12.47-fold, and 7.09-fold higher than that for Cur solution, respectively. This result shows that the bioavailability of Cur was improved by its conjugation with NPs. The half-lives of Cur-NPs improved to 9.06 hours (11.77-fold increase) and 8.48 hours (11.01-fold increase) for Cur-NPs and Fab'-Cur-NPs, respectively, compared with that of Cur solution. Fab'-Cur-NPs exhibited similar PK parameters to Cur-NPs. However, the half-life of TMAB-Cur-NPs was 1.60 hours shorter than that of Cur-NPs, likely due to Fc receptor-mediated rapid uptake into the liver and spleen as shown in Figure $8 \mathrm{~A}$ and $\mathrm{B} .{ }^{26}$ The accumulation of TMAB-Cur-NPs in the liver and spleen was significantly

Table 3 Pharmacokinetic parameters of curcumin solution (free Cur), Cur-NPs, Fab'-Cur-NPs, and TMAB-Cur-NPs in SpragueDawley rats

\begin{tabular}{|c|c|c|c|c|}
\hline Formulation & $\begin{array}{l}\text { AUC }_{0-\mathrm{t},{ }^{a}} \\
\mu \mathrm{g} / \mathrm{L} * \mathrm{~h}\end{array}$ & $t_{1 / 2}, b$ & $\mathrm{MRT}_{0-\mathrm{t}}{ }^{\mathrm{c}} \mathrm{h}$ & $C_{\max },{ }^{d} \mu g / L$ \\
\hline Free Cur & 3.23 & 0.77 & 0.22 & 9.75 \\
\hline Cur-NPs & 59.31 & 9.06 & 5.40 & 22.78 \\
\hline Fab'-Cur-NPs & 40.29 & 8.48 & 3.92 & 20.70 \\
\hline TMAB-Cur-NPs & 22.91 & 1.60 & 2.19 & 16.46 \\
\hline
\end{tabular}

Notes: ${ }^{\text {aArea }}$ under the blood concentration versus time curve $\left(\mathrm{AUC}_{0_{-} \mathrm{t}}\right)$; ${ }^{\text {bhalf-life }}$ $\left(\mathrm{t}_{1 / 2}\right)$; ${ }^{\mathrm{c}}$ mean residence time (MRT $\left.{ }_{0-\mathrm{t}}\right)$; ${ }^{\mathrm{d}}$ maximum concentration $\left(\mathrm{C}_{\max }\right)$.

Abbreviations: Cur, curcumin; Cur-NPs, curcumin nanoparticles; Fab'-Cur-NPs, fragment Fab'-modified curcumin nanoparticles; TMAB-Cur-NPs, trastuzumabmodified curcumin nanoparticles.

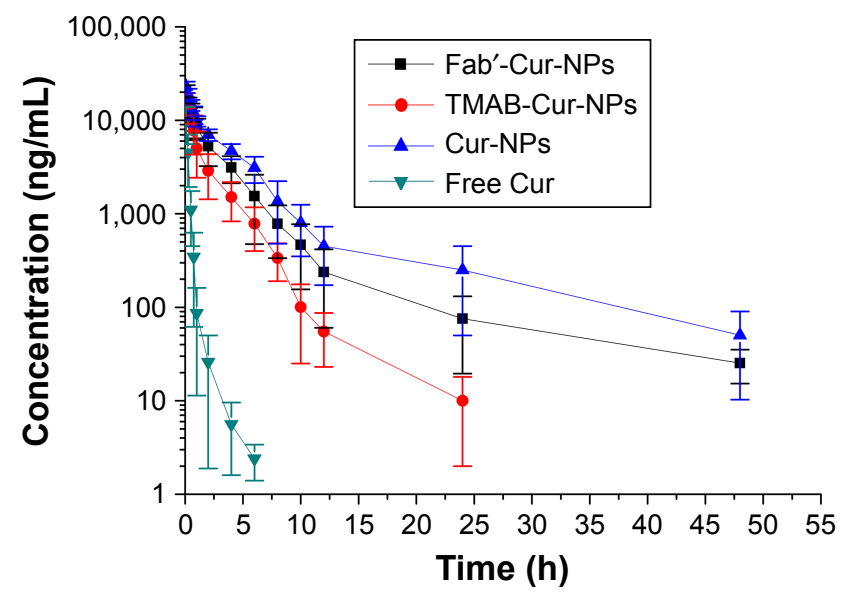

Figure 7 Plasma concentration-time profiles of curcumin after intravenous administration of curcumin solutions (free Cur), Cur-NPs, Fab'-Cur-NPs, and TMAB-CurNPs of the equivalent curcumin dose $(4 \mathrm{mg} / \mathrm{kg})$ in Sprague-Dawley rats. Values represent the mean $\pm S D(n=5)$.

Abbreviations: Cur, curcumin; Cur-NPs, curcumin nanoparticles; Fab'-Cur-NPs, fragment Fab'-modified curcumin nanoparticles; TMAB-Cur-NPs, trastuzumab-modified curcumin nanoparticles; HER2, human epidermal growth factor receptor 2.

greater $(P<0.05)$ than that of Fab'-Cur-NPs or Cur-NPs. This explains our observation that $\mathrm{AUC}_{0-\mathrm{t}}$ for Cur-NPs and $\mathrm{Fab}^{\prime}$-Cur-NPs was 2.59-fold and 1.76-fold greater than that for TMAB-Cur-NPs (Table 3). Furthermore, the uptake of Cur-NPs into the heart, kidneys, and lungs was low, with slightly greater uptake of TMAB-Cur-NPs by these tissues relative to Cur-NPs (data not shown). As shown in Figure 8C, Fab'-Cur-NP accumulation in tumor tissue was significantly greater $(P<0.05)$ than that of non-targeted NPs (Cur-NPs), and fragment $\mathrm{Fab}^{\prime}$-modified NPs have prominent targeting in vivo. The accumulation of Fab'-Cur-NPs in the tumor was greater than that of TMAB-Cur-NPs, but we observed no significant difference.

\section{Conclusion}

The novelty of this research is that Fab' fragment, which was prepared by digesting the intact antibody, was modified to a nano-vector for drug delivery to overcome the defects of TMAB conjugation. In vitro cellular uptake experiments showed that Fab'-NPs exhibited enhanced cellular accumulation compared with TMAB-NPs. PK and BD experiments in vivo demonstrated that $\mathrm{Fab}^{\prime}$-NPs expressed higher tumor permeability and longer blood circulation times. In summary, we compared the results of both in vitro and in vivo experiments performed using $\mathrm{Fab}^{\prime}$-modified nano-vector and intact antibody-modified nano-vector and demonstrated that Fab' fragment can overcome the disadvantages of intact antibody conjugation and achieve targeted drug delivery to tumors. 

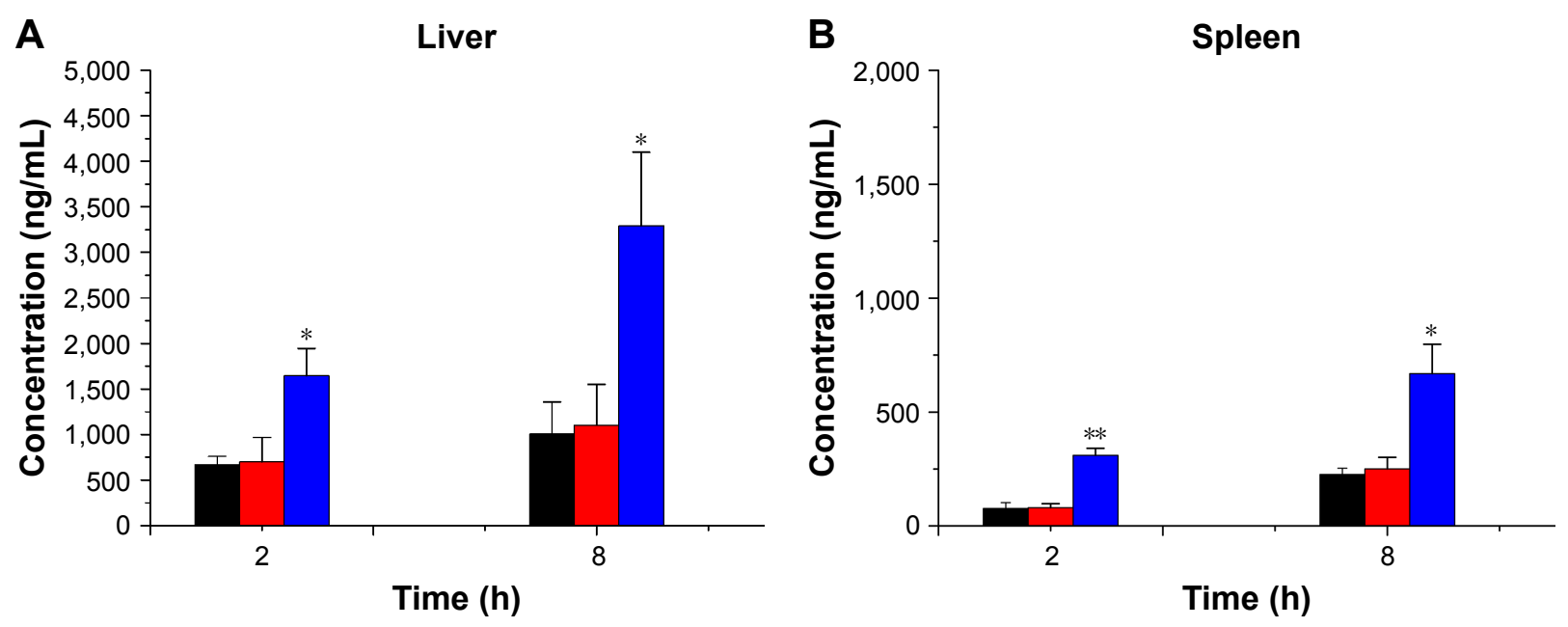

C

Tumor

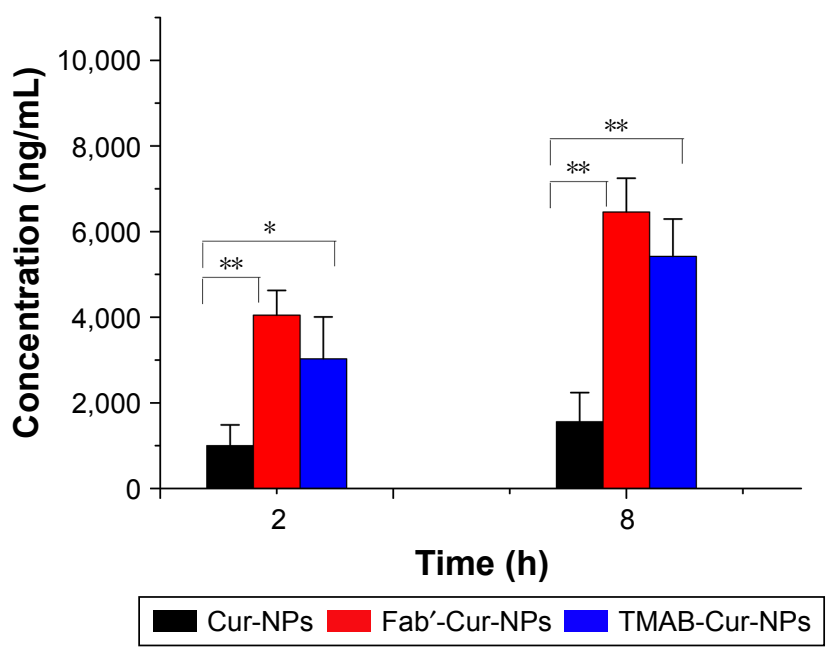

Figure 8 Biodistribution study of Cur-NPs, Fab'-Cur-NPs, and TMAB-Cur-NPs in BT-474-bearing BALB/c mice.

Notes: Mice bearing the $200 \mathrm{~cm}^{3}$ tumor were treated $24 \mathrm{~h}$ later with Cur-NPs, Fab'-Cur-NPs, or TMAB-Cur-NPs at a dose of $4 \mathrm{mg}$ curcumin/kg. Mice bearing tumor were euthanized at pre-set time points; organs were collected, and the amount of drug in tissue was measured. Data are presented as the mean \pm SD ( $=3$ ). Panels $\mathbf{A}$, B, and $\mathbf{C}$ are data collected in liver, spleen, and tumor, respectively. Significant differences in Cur-NPs and Fab'-Cur-NPs, and TMAB-Cur-NPs are marked with $*$ for $P<0.05$ and $* *$ for $P<0.01$.

Abbreviations: Cur, curcumin; Cur-NPs, curcumin nanoparticles; Fab'-Cur-NPs, fragment Fab'-modified curcumin nanoparticles; TMAB-Cur-NPs, trastuzumab-modified curcumin nanoparticles.

The results of this study could provide novel ideas for scientific research.

\section{Acknowledgments}

This study was funded by the Natural Science Foundation of Shandong Province (ZR2017LH076) and Science and Technology Project of Higher Education of Shandong Province (J16LM52). We thank Ms Liuxiang Chu for the help on the cellular uptake experiments.

\section{Disclosure}

The authors report no conflicts of interest in this work.

\section{References}

1. Ma P, Zhang X, Ni L, et al. Targeted delivery of polyamidoaminepaclitaxel conjugate functionalized with anti-human epidermal growth factor receptor 2 trastuzumab. Int J Nanomedicine. 2015;10:2173-2190.

2. Gabizon A, Goren D, Fuks Z, Barenholz Y, Dagan A, Meshorer A. Enhancement of adriamycin delivery to liver metastatic cells with increased tumoricidal effect using liposomes as drug carriers. Cancer Res. 1983;43:4730-4735.

3. Northfelt DW, Martin FJ, Working P, et al. Doxorubicin encapsulated in liposomes containing surface-bound polyethylene glycol: pharmacokinetics, tumor localization, and safety in patients with AIDS-related Kaposi's sarcoma. J Clin Pharmacol. 1996;36:55-63.

4. Shi P, Gustafson JA, MacKay JA. Genetically engineered nanocarriers for drug delivery. Int J Nanomedicine. 2014;9:1617-1626.

5. Tokuda Y. Antibodies as molecular target-based therapy: trastuzumab. Int J Clin Oncol. 2003;8:224-229. 
6. Kirpotin D, Park JW, Hong K, et al. Sterically stabilized anti-HER2 immunoliposomes: design and targeting to human breast cancer cells in vitro. Biochemistry. 1997;36:66-75.

7. Steinhauser I, Spankuch B, Strebhardt K, Langer K. Trastuzumabmodified nanoparticles: optimisation of preparation and uptake in cancer cells. Biomaterials. 2006;27:4975-4983.

8. Park JW, Hong K, Kirpotin DB, et al. Anti-HER2 immunoliposomes: enhanced efficacy attributable to targeted delivery. Clin Cancer Res. 2002; 8:1172-1181.

9. Mazzucchelli S, Colombo M, De Palma C, et al. Single-domain protein A-engineered magnetic nanoparticles: toward a universal strategy to site-specific labeling of antibodies for targeted detection of tumor cells. ACS Nano. 2010;4:5693-5702.

10. Nahta R, Esteva F. HER-2-targeted therapy: lessons learned and future directions. Clin Cancer Res. 2003;9:5078-5084.

11. Holliger P, Hudson PJ. Engineered antibody fragments and the rise of single domains. Nat Biotechnol. 2005;23:1126-1136.

12. Kenanova V, Olafsen T, Crow DM, et al. Tailoring the pharmacokinetics and positron emission tomography imaging properties of anticarcinoembryonic antigen single-chain $\mathrm{Fv}-\mathrm{Fc}$ antibody fragments. Cancer Res. 2005;65(2):622-631.

13. Béduneau A, Saulnier P, Hindré F, Clavreul A, Leroux JC, Benoit JP. Design of targeted lipid nanocapsules by conjugation of whole antibodies and antibody Fab' fragments. Biomaterials. 2007;28:4978-4990.

14. Fiandra L, Mazzucchelli S, De Palma C, et al. Assessing the in vivo targeting efficiency of multifunctional nanoconstructs bearing antibodyderived ligands. ACS Nano. 2013;7:6092-6102.

15. Chithrani BD, Warren CWC. Elucidating the mechanism of cellular uptake and removal of protein-coated gold nanoparticles of different sizes and shapes. Nano Lett. 2007;7:1542-1550.

16. Gao H, Shi W, Freund LB. Mechanics of receptor-mediated endocytosis. Proc Natl Acad Sci US A. 2005;102:9469-9474.
17. Koning $\mathrm{G}$, Morselt $\mathrm{H}$, Gorter A, et al. Interaction of differently designed immunoliposomes with colon cancer cells and Kupffer cells. An in vitro comparison. Pharm Res. 2003;20:1249-1257.

18. Freise AC, Wu AM. In vivo imaging with antibodies and engineered fragments. Mol Immunol. 2015;67:142-152.

19. Cheng WW, Allen TM. Targeted delivery of anti-CD19 liposomal doxorubicin in B-cell lymphoma: a comparison of whole monoclonal antibody, Fab' fragments and single chain Fv. J Control Release. 2008;126: 50-58.

20. Cartiera MS, Ferreira EC, Caputo C, Egan ME, Caplan MJ, Saltzman WM. Partial correction of cystic fibrosis defects with PLGA nanoparticles encapsulating curcumin. Mol Pharm. 2010;7:86-93.

21. Mathew A, Fukuda T, Nagaoka Y, et al. Curcumin loaded-PLGA nanoparticles conjugated with Tet-1 peptide for potential use in Alzheimer's disease. PLoS One. 2012;7:e32616.

22. Lee CY, Ooi IH. Preparation of temozolomide-loaded nanoparticles for glioblastoma multiforme targeting-ideal versus reality. Pharmaceuticals (Basel). 2016;9:pii:E54.

23. Muntimadugu E, Dhommati R, Jain A, Challa VG, Shaheen M, Khan W. Intranasal delivery of nanoparticle encapsulated tarenflurbil: a potential brain targeting strategy for Alzheimer's disease. Eur J Pharm Sci. 2016;92:224-234

24. Iden $\mathrm{D}$, Allen $\mathrm{T}$. In vitro and in vivo comparison of immunoliposomes made by conventional coupling techniques with those made by a new post-insertion approach. Biochim Biophys Acta. 2001;1513:207-216.

25. Pahuja R, Seth K, Shukla A, et al. Trans-blood brain barrier delivery of dopamine-loaded nanoparticles reverses functional deficits in parkinsonian rats. ACS Nano. 2015;9:4850-4871.

26. Maruyama K, Takahashi N, Tagawa T, et al. Immunoliposomes bearing polyethyleneglycol-coupled Fab' fragment show prolonged circulation time and high extravasation into targeted solid tumors in vivo. FEBS Lett. 1997;413:177-180.
International Journal of Nanomedicine

\section{Publish your work in this journal}

The International Journal of Nanomedicine is an international, peerreviewed journal focusing on the application of nanotechnology in diagnostics, therapeutics, and drug delivery systems throughout the biomedical field. This journal is indexed on PubMed Central, MedLine, CAS, SciSearch ${ }^{\circledR}$, Current Contents ${ }^{\circledR} /$ Clinical Medicine,

\section{Dovepress}

Journal Citation Reports/Science Edition, EMBase, Scopus and the Elsevier Bibliographic databases. The manuscript management system is completely online and includes a very quick and fair peer-review system, which is all easy to use. Visit http://www.dovepress.com/ testimonials.php to read real quotes from published authors. 